\title{
Effects of pentoxifylline treatment before freezing on motility, viability and acrosome status of poor quality human spermatozoa cryopreserved by the liquid nitrogen vapor method
}

\section{S.C. Esteves ${ }^{1,2}$, \\ D.M. Spaine ${ }^{1}$} and A.P. Cedenho ${ }^{1}$

\author{
${ }^{1}$ Laboratório de Reprodução Humana, Disciplina de Urologia, \\ Escola Paulista de Medicina, Universidade Federal de São Paulo, São Paulo, SP, Brasil \\ ${ }^{2}$ ANDROFERT - Centro de Referência em Reprodução Masculina, Campinas, SP, Brasil
}

\section{Correspondence \\ S.C. Esteves \\ ANDROFERT - Centro de Referência \\ em Reprodução Masculina \\ Av. Dr. Heitor Penteado, 1464 \\ 13075-460 Campinas, SP \\ Brasil \\ Fax: +55-19-3294-6992 \\ E-mail: s.esteves@androfert.com.br}

Part of the data were presented by S.C. Esteves as a Doctoral thesis

to the Federal University of

São Paulo, Brazil.

Publication supported by Institutional Funding for Research.

Received September 29, 2006 Accepted May 8, 2007

\begin{abstract}
The objective of the present study was to investigate the effects of the direct addition of pentoxifylline (PF) to the ejaculates of men with poor sperm quality before freezing on post-thaw sperm motility, viability, acrosome integrity, and agonist-induced acrosome reaction. Semen specimens from 16 infertile men with impaired sperm count and motility (oligoasthenozoospermia) were divided into two equal aliquots: one received no treatment (control) while the other was incubated with $5 \mathrm{mM} \mathrm{PF}$ (treated). Both aliquots were cryopreserved by the liquid nitrogen vapor method. Motility was assessed according to WHO criteria. Acrosome integrity and spontaneous and calcium ionophore-induced acrosome reactions were assessed with fluorescein isothiocyanate-conjugated peanut agglutinin combined with a supra-vital dye (Hoechst-33258). Cryopreservation impaired sperm motility (percentage reduction: 87.4 (interquartile range, IQ: 70.392.9) vs 89.1 (IQ: 72.7-96.0\%)), viability (25.9 (IQ: 22.2-29.7) vs 25.6 (IQ: 19.7-40.3\%)) and acrosome integrity (18.9 (IQ: 5.4-38.9) vs 26.8 (IQ: $0.0-45.2 \%)$ ) to the same extent in both treated and control aliquots. However, PF treatment before freezing improved the acrosome reaction to ionophore challenge test scores in cryopreserved spermatozoa (9.7 (IQ: 6.6-19.7) vs 4.8 (IQ: 0.5-6.8\%); P = 0.002). These data show that pre-freeze treatment of poor quality human sperm with pentoxifylline did not improve post-thaw motility or viability nor did it prevent acrosomal loss during the freeze-thaw process. However, PF, as used, improved the ability of thawed spermatozoa to undergo the acrosome reaction in response to calcium ionophore. The present data indicate that treatment of poor quality human sperm with PF may enhance post-thaw sperm fertilizing ability.
\end{abstract}

Key words - Acrosome

- Cryopreservation

- Human spermatozoa

- Ionophore

- Pentoxifylline

- Oligoasthenozoospermia 


\section{Introduction}

Cryopreservation impairs sperm fertilizing ability by decreasing sperm motility and motion characteristics, penetration into the cervical mucus and into zona-free hamster eggs, viability, membrane integrity and acrosome structure, and activity of acrosome protease and acrosin (1-4). Pentoxifylline (PF), a phosphodiesterase inhibitor, has been used to stimulate the motility and fertilizing capacity of human sperm (5-8). PF inhibits cyclic adenosine monophosphate (cAMP) phosphodiesterase, thus increasing intracellular cAMP concentration $(5,7)$ and tyrosine-phosphorylation at the tail level (8). cAMP has a role in sperm kinematics and in the acrosome reaction second-messenger system $(7,9)$. Treatments that increase intracellular cAMP concentrations often cause an increase in sperm motility and kinematics as well as in the agonist-induced acrosome reaction (9) and fertilization rates (5,6). Also, it has been postulated that PF has the ability to scavenge reactive oxygen radicals $(10,11)$.

It has been reported that pre-freeze sperm treatment with PF decreases acrosome loss during the freeze-thaw process and increases the post-thaw agonist-induced acrosome reaction rate in normal semen (12). This strategy may be clinically useful because it can improve the fertilizing potential of cryopreserved human semen. One of the major advantages of sperm banking is the fertility preservation in cancer patients prior to potential gonadotoxic treatments, such as radiotherapy and chemotherapy. Although specimens of cancer patients freeze as effectively as those from normal men, the semen quality at the time of cryopreservation is often poor in such individuals, especially in testicular cancer patients (13). In the present study, we investigated if the beneficial effects of the addition of PF directly to the seminal plasma before freezing, as seen in normal human semen (12), can also be demonstrated in poor quality specimens, such as those obtained from infertile men with decreased sperm count and motility (oligoasthenozoospermia).

\section{Patients and Methods}

\section{Chemicals}

TEST yolk-buffer freezing medium, sperm-washing medium (modified BiggersWhitten-Whittingam; BWW) and Dulbecco's phosphate-buffered saline (PBS) were purchased from Irvine Scientific (Santa Ana, CA, USA). Fluorescein isothiocyanate-conjugated peanut agglutinin (FITC-PNA), calcium ionophore A23187, PF, and bis-benzimide (Hoechst-33258) were obtained from Sigma (St. Louis, MO, USA).

A stock solution of FITC-PNA was prepared by dissolving 2 mg FITC-PNA in 1 $\mathrm{mL}$ PBS and stored in $600-\mu \mathrm{L}$ aliquots at $-20^{\circ} \mathrm{C}$. Hoechst-33258 was prepared by dissolving $1 \mathrm{mg} \mathrm{H} 33258$ in $1 \mathrm{~mL}$ PBS and stored in $10-\mu \mathrm{L}$ aliquots at $-20^{\circ} \mathrm{C}$.

\section{Semen collection, sperm processing and cryopreservation procedures}

Sperm specimens were obtained by masturbation into sterile specimen cups from men seeking infertility evaluation at a University-based hospital. All subjects were asked to abstain from ejaculation from 2 to 3 days before collection. After liquefaction at $37^{\circ} \mathrm{C}$, a small aliquot was removed from each specimen and the sperm parameters were determined according to World Health Organization (WHO) guidelines (14). A total of 16 oligoasthenozoospermic semen samples were selected according to WHO criteria for sperm count of $<20 \times 10^{6} / \mathrm{mL}$ and total motility of $<50 \%$. The etiology of male infertility was not taken into consideration in this study. The median values of sperm count $($ million $/ \mathrm{mL})$, percent motility and seminal plasma leukocytes, as assessed by the Endtz test (14), were 12.0 (25-75\% inter- 
quartile range: $9.0-14.0), 40.5$ (25-75\% interquartile range: $30.0-59.0$ ), and 0.2 (interquartile range: 0.1-0.4), respectively. Informed consent from all subjects included in the study and approval by the Institution Ethics Committee were obtained. The experimental design and methods were identical to those reported in a previous study (12). Briefly, after initial analysis each specimen was divided into two equal aliquots. The first aliquot received no treatment (control) and the second was treated by adding a 10$\mathrm{mM}$ PF solution directly to the liquefied ejaculate $(1: 1, \mathrm{v} / \mathrm{v})$. The final concentration of PF in the mixture was $5 \mathrm{mM}$. Control and treated aliquots were incubated for $30 \mathrm{~min}$ at $37^{\circ} \mathrm{C}$ and then evaluated for percent and progressive motility according to $\mathrm{WHO}$ criteria (14), and for acrosome integrity $(12,15)$.

Control and treated specimens were then cryopreserved by the liquid nitrogen vapor method (12). TEST-yolk buffer with glycerol was used as a freezing agent for cryopreservation (15). Briefly, an aliquot of the freezing medium equal to $25 \%$ of the original specimen volume was added to the specimen. This process was repeated until the ratio of freezing medium to ejaculate was $1: 1$. Cryovials were loaded with $1 \mathrm{~mL}$ freezing medium/ejaculate mixture, and kept at $-20^{\circ} \mathrm{C}$ for $8 \mathrm{~min}$. Next, the cryovials were exposed to liquid nitrogen vapor at $-79^{\circ} \mathrm{C}$ for $2 \mathrm{~h}$ before being plunged into liquid nitrogen for storage at $-196^{\circ} \mathrm{C}$. After at least $48 \mathrm{~h}$ in liquid nitrogen, the samples were thawed at room temperature for $5 \mathrm{~min}$ and transferred to a $37^{\circ} \mathrm{C}$ incubator for $20 \mathrm{~min}$ (4). In order to remove the cryomedium, the thawed specimens were diluted with BWW medium at a ratio of 1 volume of freezing medium/ejaculate to 3 volumes of medium and washed by centrifugation at $300 \mathrm{~g}$ for $7 \mathrm{~min}$. The supernatant was removed and the pellet resuspended in $0.7 \mathrm{~mL} \mathrm{BWW}$ medium with $0.3 \%$ human serum albumin. A small aliquot (0.1 $\mathrm{mL}$ ) was removed from each washed specimen for the assessment of percent motility, progressive motility and the frequency of spontaneous acrosome reaction.

\section{Sperm incubation under capacitating conditions and ionophore challenge test}

After thawing, the motile sperm population from each specimen was isolated by the swim-up method from a washed sperm preparation (15). The motile spermatozoa were then capacitated for $3 \mathrm{~h}$ by incubation in BWW medium with 3\% human serum albumin at $37^{\circ} \mathrm{C}$ in a $5 \% \mathrm{CO}_{2}$ atmosphere (4). After capacitation the specimens were divided into two equal aliquots: one was incubated with $2.5 \mu \mathrm{mol} / \mathrm{L}$ calcium ionophore A23187 solution, and the other was treated with dimethylsulfoxide $(10 \%, \mathrm{v} / \mathrm{v})$ solution to serve as a control (12). Both aliquots were then incubated at $37^{\circ} \mathrm{C}$ for $1 \mathrm{~h}$ under the same experimental conditions.

Assessment of viability, acrosome status and ionophore-induced acrosome reaction

Acrosome status was assessed with FITCPNA in combination with the nuclear stain bis-benzimide to monitor viability. Viability and acrosome status were assessed simultaneously by mixing $100 \mu \mathrm{L}$ of sperm specimens with $100 \mu \mathrm{L}$ of a $2-\mu \mathrm{g} / \mathrm{mL}$ Hoechst33258 solution (12). The mixture was incubated for 10 min in the dark. Excess stain was removed from the spermatozoa by dilution with PBS and centrifugation at $300 \mathrm{~g}$ for $5 \mathrm{~min}$, and the pellet was resuspended in 100 $\mu \mathrm{L}$ BWW. A $20-\mu \mathrm{L}$ aliquot of this solution was smeared on a microscope slide and allowed to dry in the dark at room temperature. Air-dried slides were immersed in icecold methanol for $30 \mathrm{~s}$ to permeabilize the sperm membranes. The fixed smears were immersed in a solution of $40 \mu \mathrm{g} / \mathrm{mL}$ FITCPNA for $20 \mathrm{~min}$, and washed gently in PBS to remove excess label.

A fluorescence microscope (Eclipse E600, Nikon, Tokyo, Japan) equipped with 
an epi-illumination module and a mercury ultraviolet source was used to examine the slides at 1000X magnification. The B2A filter cube was used for FITC-PNA, which fluoresces apple-green, and the UV2A cube for Hoechst-33258, which fluoresces a bright medium blue. Hoechst-33258 stains the nuclei of damaged cells (dead spermatozoa), which show a bright blue-white fluorescence, and is excluded from viable cells (live spermatozoa), which show a pale blue fluorescence. The same spermatozoon was examined for FITC-PNA labeling and for Hoechst33258 staining by interchanging the two filters (12).

\section{Staining patterns showing sperm viability and acrosome status}

In viable spermatozoa, the sperm head showed a pale-blue fluorescence, and in dead spermatozoa, the sperm head showed a bright blue-white fluorescence. In an intact acrosome, the acrosomal region of the sperm head exhibited a uniform apple-green fluorescence, and in a reacted acrosome, only the equatorial segment of the acrosome was stained. A total of 200 spermatozoa per sample were scored. The calculated frequency of spermatozoa with intact and reacted acrosomes was based on viable cells only. The acrosome reaction to ionophore challenge (ARIC) scores was calculated by subtracting the frequency of acrosome reaction occurrence following ionophore challenge from the frequency of occurrence before it.

\section{Statistical analysis}

On the basis of previously published data showing that the average percent decrease in sperm motility, viability and acrosome integrity after the freeze-thawing process is about $15 \%(2,4,12,13,15,16)$, and that in vitro sperm treatment with $\mathrm{PF}$ increases sperm motility and acrosome response to ionophore challenge from 10 to $20 \%(12,17$,
18), we expected that similar results would be achieved with the experimental conditions used in the present study. Assuming a level of significance of 0.05 and a power of $80 \%$, it was calculated that 15 subjects were needed in each experimental group to detect differences of $\geq 10 \%$ in the main outcome measures (sperm motility, viability, acrosome integrity, and acrosome reaction in response to ionophore challenge). Data were assessed for homogeneity and normality by the Kolgomorov-Smirnov test. Statistical differences in sperm motility, viability and acrosome status between control and treated specimens before or after cryopreservation were assessed by the Wilcoxon signed-rank test. Data are reported as median and interquartile range. The level of significance was set at $\mathrm{P}<0.05$. Statistical analysis was performed using the SAS statistical software package (Cary, NC, USA).

\section{Results}

Pre-freeze and post-thaw sperm motility and viability in control and PF-treated specimens are compared in Table 1. Before freezing, PF treatment significantly increased both the percentage of motile spermatozoa $(\mathrm{P}<$ $0.01)$ and the percentage of spermatozoa exhibiting progressive motility $(\mathrm{P}=0.006)$. However, PF treatment did not alter prefreeze sperm viability. The freeze-thaw process significantly decreased sperm motility $(\mathrm{P}<0.001)$, progressive motility $(\mathrm{P}<0.001)$ and viability $(\mathrm{P}<0.001)$ in both control and treated specimens. The percent change in these parameters from before freezing to after thawing was not statistically different between treated and non-treated groups.

Percentages of viable spermatozoa with intact acrosomes in control and PF groups before and after cryopreservation are shown in Table 1. Acrosome integrity did not differ between control and treated groups before freezing. The freeze-thaw process significantly decreased the percentages of live 
sperm with intact acrosomes in both the control and PF groups $(\mathrm{P}<0.001$; Table 1$)$. The percentage of spermatozoa that lost acrosomal contents during freezing and thawing, assessed by subtracting the percentage of viable-intact acrosome spermatozoa postthaw from the pre-freezing percentage, was 18.9 and $26.8 \%$ in the control and PF groups, respectively (Table 1 ). However, the differences were not statistically significant.

The effects of calcium ionophore on the acrosome status of thawed viable spermatozoa of the control and PF groups are shown in Table 2. After thawing, the percentage of live spermatozoa exhibiting a spontaneous acrosome reaction in control and treated specimens was 43.9 and $38.0 \%$, respectively (Table 2). Again the differences were not statistically significant.

The ARIC was calculated by subtracting the frequency of acrosome reaction occurrence after ionophore challenge from the frequency of occurrence before it. ARIC scores were significantly increased in both the control $(\mathrm{P}=0.02)$ and $\mathrm{PF}$ groups $(\mathrm{P}<$ $0.001)$. However, ARIC scores were significantly higher in the PF-treated group (median: $9.7 \%$ (25-75\% interquartile range: $6.6-$ 19.7)) than in control (median: $4.8 \%$ (25$75 \%$ interquartile range: $0.5-6.8 ; \mathrm{P}=0.002)$ ).

We observed that ARIC scores were higher than $10 \%$ in $50 \%$ (8 of 16) of the specimens in the $\mathrm{PF}$ group. In the remaining specimens, ARIC scores were between 5 and $10 \%$ in $6(6 / 8)$, and $<5 \%$ in only 2 specimens (Figure 1). In the control group, only 19\% (3 of 16) of the specimens had ARIC scores $>10 \%$. In the remaining 13 specimens, five exhibited ARIC scores between 5 and $10 \%$, and 8 scores $<5 \%$. When an individual specimen was compared to its own control, pre-freeze PF treatment was found to improve the ARIC scores in $81.2 \%$ of the specimens (13/16; Figure 1). Interestingly, pre-freeze PF treatment improved ARIC scores to $>10 \%$ in all but $1(4 / 5)$ control specimens with scores between 5
Table 1. Effect of pre-freeze treatment with pentoxifylline on motility, viability and acrosome integrity of sperm from 16 oligoasthenozoospermic men before and after cryopreservation.

\begin{tabular}{|c|c|c|}
\hline Sperm parameters & Control & Pentoxifylline \\
\hline \multicolumn{3}{|l|}{ Percent motility } \\
\hline Pre-freeze & $40.5(30.0-59.0)$ & $55.5(41.5-62.5)^{\star}$ \\
\hline Post-thaw & $3.5(2.5-10.5)$ & $5.0(2.5-12.0)$ \\
\hline Percent change ${ }^{a}$ & $87.4(70.3-92.9)$ & $89.1(72.7-96.0)$ \\
\hline $\mathrm{P}$ value ${ }^{\mathrm{b}}$ & $<0.001$ & $<0.001$ \\
\hline \multicolumn{3}{|c|}{ Progressive motility (\%grades $a+b$ ) } \\
\hline Pre-freeze & $35.5(24.0-58.0)$ & $47.0(34.5-59.5)^{\star}$ \\
\hline Post-thaw & $1.5(0.0-5.0)$ & $2.0(0.0-6.0)$ \\
\hline Percent change ${ }^{a}$ & $94.5(74.1-99.9)$ & $94.4(84.1-100.0)$ \\
\hline$P$ value ${ }^{b}$ & $<0.001$ & $<0.001$ \\
\hline \multicolumn{3}{|l|}{ Sperm viability (\%) } \\
\hline Pre-freeze & $76.2(73.0-90.0)$ & $74.6(70.6-92.1)$ \\
\hline Post-thaw & $54.6(48.0-61.2)$ & $50.7(44.7-59.2)$ \\
\hline Percent change ${ }^{a}$ & $25.9(22.2-29.7)$ & $25.6(19.7-40.3)$ \\
\hline $\mathrm{P}$ value $^{\mathrm{b}}$ & $<0.001$ & $<0.001$ \\
\hline \multicolumn{3}{|c|}{ Acrosome integrity (\%) } \\
\hline Pre-freeze & $79.2(67.7-82.7)$ & $76.2(73.2-81.5)$ \\
\hline Post-thaw & $62.8(52.9-80.2)$ & $58.0(45.9-71.2)$ \\
\hline Percent change $^{a}$ & $18.9(5.4-38.9)$ & $26.8(0.0-45.2)$ \\
\hline$P$ value ${ }^{b}$ & $<0.001$ & $<0.001$ \\
\hline
\end{tabular}

Data are reported as median with interquartile range in parentheses. Progressive motility = grade a (spermatozoa exhibiting fast progression) and grade b (spermatozoa exhibiting slow progression) according to WHO criteria.

aPercent change calculated as median of individual changes; bP value comparing prefreeze to post-thaw (Wilcoxon signed-rank test). ${ }^{*} P<0.05$ compared to control (Wilcoxon signed-rank test).

Table 2. Effects of pre-freeze treatment with pentoxifylline on post-thaw calcium ionophore-induced acrosome reaction in sperm from 16 oligoasthenozoospermic men.

\begin{tabular}{lcc}
\hline $\begin{array}{l}\text { Post-thaw frequency of live } \\
\text { spermatozoa with reacted } \\
\text { acrosomes (\%) }\end{array}$ & Control & Pentoxifylline \\
\hline Before ionophore challenge & $43.9(27.2-54.5)$ & $38.0(30.1-51.2)$ \\
After ionophore challenge & $45.6(25.7-59.1)$ & $52.7(38.5-67.4)^{*}$ \\
Percent change & $-11.8(-(32.0-2.9))$ & $-30.2(-(40.8-18.5))^{*}$ \\
${\text { ARIC score }(\%)^{*}}_{P}$ & $4.8(0.5-6.8)$ & $9.7(6.6-19.7)$ \\
P value & $<0.001$ & $<0.001$
\end{tabular}

Data are reported as median with interquartile range in parentheses. Acrosome reaction in response to ionophore challenge (ARIC) score was calculated by subtracting the frequency of acrosome reaction occurrence after ionophore challenge from the frequency of occurrence before it.

apercent change calculated as median of individual changes; bP value comparing frequency of live spermatozoa with reacted acrosomes before and after ionophore challenge (Wilcoxon signed-rank test). ${ }^{*} \mathrm{P}<0.05$ compared to control (Wilcoxon signed-rank test). 
and $10 \%$. Similarly, pre-freeze PF treatment improved ARIC scores to $>5 \%$ in all but 1 (7/8) control specimens with scores $<5 \%$. Only 3 specimens were refractive to $P F$ treatment (Figure 1).

\section{Discussion}

At a concentration of $5 \mathrm{mM} P \mathrm{PF}$ has oxygen-free radical scavenging capacities by reducing the superoxide release from human spermatozoa following phorbol myristate acetate (11). Higher PF concentrations, however, may be detrimental to membrane integrity (19). Normal sperm incubated with 5 $\mathrm{mM}$ PF prior to cryopreservation are protected from the deleterious effects of the freeze-thaw process (12). Based on these findings, PF may be used as a supplement to the cryomedia currently available. In this study, we examined if the benefits of PF for freezing normal sperm can be extrapolated to poor quality sperm obtained from oligoasthenozoospermic men. Oligoasthenozoospermia is often seen in cancer patients, who are the main patient population seeking sperm banking. Our results indicated that

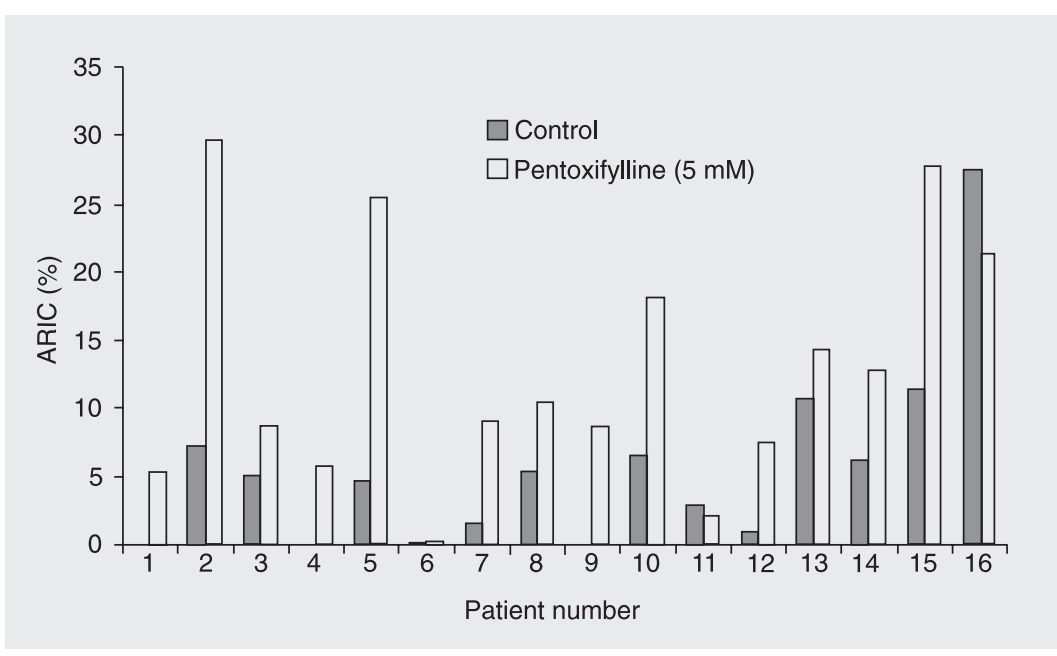

Figure 1. Effects of pre-freeze pentoxifylline treatment on post-thaw acrosome reaction to ionophore challenge (ARIC) in sperm from 16 oligoasthenozoospermic men. Bars represent ARIC scores for each individual: control (filled bars) and $5 \mathrm{mM}$ pentoxifylline-treated (open bars). Filled bars are not seen in controls 1,4 , and 9 because ARIC scores were equal to zero (post-thaw live spermatozoa in these specimens were refractive to stimulation with calcium ionophore). the cryopreservation process was detrimental to sperm motility and viability, irrespective of whether or not spermatozoa had been treated with PF before freezing, corroborating previous reports (12). Others have demonstrated an enhancement in post-thaw sperm motility when PF was added prior to cryopreservation $(17,18)$. It is possible that at the concentration used in our study, post-thaw spermatozoa were unable to respond effectively to PF in terms of motility, either because of membrane damage caused by cryopreservation, or because they may be sensitized and require different concentrations for stimulation. Moreover, we observed a significant decrease in the post-thaw percentage of viable spermatozoa with intact acrosomes in both control and PF-treated specimens. In normal semen, a PF-induced protective effect on acrosome loss during the freeze-thaw process was observed (12). In the present study, we evaluated poor quality semen, and it is possible that normal semen would resist cryoinjury better than semen obtained from oligoasthenozoospermic men. We observed that prefreeze treatment of sperm from such individuals with PF improved the ability of cryo-thawed spermatozoa to undergo the acrosome reaction in response to stimuli. Interestingly, the ability of cryo-thawed sperm from abnormal specimens to respond to ionophore challenge appears to be lower than that of normal sperm, irrespectively of treatment with PF before freezing (12). In our study, post-thaw acrosome reaction stimulated by calcium ionophore was 11.8 and $30.2 \%$ for control and treated specimens, respectively, while values of 34.8 and $53.3 \%$ have been reported for normal men (12). A possible explanation for these differences is that $\mathrm{PF}$, as used, may afford cryoprotection in normal semen while a different concentration is necessary to neutralize excessive reactive oxygen species (ROS) emanating from the already compromised spermatozoa from oligoasthenozoospermic individuals. To our 
knowledge, this is the first study to suggest that the benefits of using PF for freezing human sperm may be related to sperm quality.

Although we did not examine ROS levels in the seminal plasma, it has been shown that ROS levels are significantly higher, and seminal plasma antioxidant capacity is significantly reduced in poor quality sperm, independent of the clinical diagnosis of male infertility (20). We speculate that cryopreserved spermatozoa from men with defective spermatogenesis may have higher levels of ROS than those from normal men. Consequently, peroxidation of unsaturated fatty acids in the acrosomal membranes may be more extended, thus decreasing the response to calcium influx signals that trigger the acrosome reaction.

Sperm function tests such as the acrosome reaction are better fertility predictors than traditional semen parameters (21-28). The mechanism of the acrosome reaction induced by ionophores differs from the physiological acrosome reaction induced by the zona pellucida (22). While the physiological acrosome reaction involves activation of several signal transduction pathways, the ionophore-induced acrosome reaction mainly involves a chemical effect on calcium influx that partially reflects the physiological acrosome reaction process (22). We did not test other physiological agonists on the acrosome reaction, such as progesterone or recombinant zona pellucida. Instead, we elected to use the ionophore-induced acrosome reaction because it has been extensively validated as a sperm function test with excellent predictive values for outcomes in assisted reproduction (23-28). Using an ARIC cutoff of $10 \%$, the sensitivity of the test in predicting subfertility was $54 \%$, with a positive predictive value of $64 \%$ and specificity of $85 \%$ (24-26). In the present study, ARIC scores were higher than $10 \%$ in $50 \%$ of the cryopreserved PF-treated specimens, and in only $19 \%$ of the controls. In normal men, ARIC scores higher than $10 \%$ were seen in 80 and $60 \%$ of the cryo-thawed PF-treated and control specimens, respectively (12). We observed that PF improved ARIC scores in $81.2 \%$ of our subjects, whereas improvement in all individuals was seen in normal semen (12).

We have shown that pre-freeze treatment of poor quality human sperm with PF improved the ability of thawed spermatozoa to undergo the acrosome reaction in response to calcium ionophore. Further investigation is needed to determine whether treatment of poor quality human sperm with PF before freezing improves fertilization and pregnancy outcomes in assisted reproduction.

\section{References}

1. Critser JK, Arneson BW, Aaker DV, Huse-Benda AR, Ball GD. Cryopreservation of human spermatozoa. II. Postthaw chronology of motility and of zona-free hamster ova penetration. Fertil Steril 1987; 47: 980-984.

2. Esteves SC, Sharma RK, Thomas AJ Jr, Agarwal A. Suitability of the hypo-osmotic swelling test for assessing the viability of cryopreserved sperm. Fertil Steril 1996; 66: 798-804.

3. McLaughlin EA, Ford WC, Hull MG. Motility characteristics and membrane integrity of cryopreserved human spermatozoa. J Reprod Fertil 1992; 95: 527-534.

4. Esteves SC, Sharma RK, Thomas AJ Jr, Agarwal A. Effect of in vitro incubation on spontaneous acrosome reaction in fresh and cryopreserved human spermatozoa. Int J Fertil Womens Med 1998; 43:
235-242.

5. Yovich JL. Pentoxifylline: actions and applications in assisted reproduction. Hum Reprod 1993; 8: 1786-1791.

6. Kovacic B, Vlaisavljevic V, Reljic M. Clinical use of pentoxifylline for activation of immotile testicular sperm before ICSI in patients with azoospermia. J Androl 2006; 27: 45-52.

7. Tesarik J, Mendoza C, Carreras A. Effects of phosphodiesterase inhibitors caffeine and pentoxifylline on spontaneous and stimulusinduced acrosome reactions in human sperm. Fertil Steril 1992; 58: 1185-1190.

8. Yunes R, Fernandez P, Doncel GF, Acosta AA. Cyclic nucleotide phosphodiesterase inhibition increases tyrosine phosphorylation and hyper motility in normal and pathological human spermatozoa. 
Biocell 2005; 29: 287-293.

9. De Jonge CJ, Han HL, Lawrie H, Mack SR, Zaneveld LJ. Modulation of the human sperm acrosome reaction by effectors of the adenylate cyclase/cyclic AMP second-messenger pathway. J Exp Zool 1991; 258: $113-125$.

10. Freitas JP, Filipe PM. Pentoxifylline. A hydroxyl radical scavenger. Biol Trace Elem Res 1995; 47: 307-311.

11. McKinney KA, Lewis SE, Thompson W. The effects of pentoxifylline on the generation of reactive oxygen species and lipid peroxidation in human spermatozoa. Andrologia 1996; 28: 15-20.

12. Esteves SC, Sharma RK, Thomas AJ Jr, Agarwal A. Cryopreservation of human spermatozoa with pentoxifylline improves the postthaw agonist-induced acrosome reaction rate. Hum Reprod 1998; 13: 3384-3389.

13. Agarwal A, Tolentino MV Jr, Sidhu RS, Ayzman I, Lee JC, Thomas AJ Jr, et al. Effect of cryopreservation on semen quality in patients with testicular cancer. Urology 1995; 46: 382-389.

14. World Health Organization. WHO laboratory manual for the examination of human semen and semen-cervical mucus interaction. 4th edn. Cambridge: The Press Syndicate of the University of Cambridge; 2007.

15. Esteves SC, Sharma RK, Thomas AJ Jr, Agarwal A. Improvement in motion characteristics and acrosome status in cryopreserved human spermatozoa by swim-up processing before freezing. Hum Reprod 2000; 15: 2173-2179.

16. Paul M, Sumpter JP, Lindsay KS. Action of pentoxifylline directly on semen. Hum Reprod 1995; 10: 354-359.

17. Brennan AP, Holden CA. Pentoxifylline-supplemented cryoprotectant improves human sperm motility after cryopreservation. Hum Reprod 1995; 10: 2308-2312.

18. Bell M, Wang R, Hellstrom WJ, Sikka SC. Effect of cryoprotective additives and cryopreservation protocol on sperm membrane lipid peroxidation and recovery of motile human sperm. J Androl 1993; 14: $472-478$.
19. Tournaye H, Janssens $R$, Verheyen G, Devroey $P$, Van SA. In vitro fertilization in couples with previous fertilization failure using sperm incubated with pentoxifylline and 2-deoxyadenosine. Fertil Steril 1994; 62: 574-579.

20. Pasqualotto FF, Sharma RK, Nelson DR, Thomas AJ, Agarwal A. Relationship between oxidative stress, semen characteristics, and clinical diagnosis in men undergoing infertility investigation. Fertil Steril 2000; 73: 459-464.

21. Liu DY, Baker HW. Disordered acrosome reaction of spermatozoa bound to the zona pellucida: a newly discovered sperm defect causing infertility with reduced sperm-zona pellucida penetration and reduced fertilization in vitro. Hum Reprod 1994; 9: 1694-1700.

22. Breitbart $\mathrm{H}$, Spungin B. The biochemistry of the acrosome reaction. Mol Hum Reprod 1997; 3: 195-202.

23. Yovich JM, Edirisinghe WR, Yovich JL. Use of the acrosome reaction to ionophore challenge test in managing patients in an assisted reproduction program: a prospective, double-blind, randomized controlled study. Fertil Steril 1994; 61: 902-910.

24. Calvo L, Dennison-Lagos L, Banks SM, Dorfmann A, Thorsell LP, Bustillo $\mathrm{M}$, et al. Acrosome reaction inducibility predicts fertilization success at in-vitro fertilization. Hum Reprod 1994; 9: 1880-1886.

25. Henkel R, Muller C, Miska W, Gips H, Schill WB. Determination of the acrosome reaction in human spermatozoa is predictive of fertilization in vitro. Hum Reprod 1993; 8: 2128-2132.

26. Cummins JM, Pember SM, Jequier AM, Yovich JL, Hartmann PE. A test of the human sperm acrosome reaction following ionophore challenge. Relationship to fertility and other seminal parameters. $J$ Androl 1991; 12: 98-103.

27. Liu DY, Baker HW. Defective sperm-zona pellucida interaction: a major cause of failure of fertilization in clinical in-vitro fertilization. Hum Reprod 2000; 15: 702-708.

28. Katsuki T, Hara T, Ueda K, Tanaka J, Ohama K. Prediction of outcomes of assisted reproduction treatment using the calcium ionophore-induced acrosome reaction. Hum Reprod 2005; 20: 469-475. 